\title{
Path dependent volatility
}

\author{
Paolo Foschi And Andrea Pascucci \\ Dipartimento di Matematica, Università di Bologna *
}

\begin{abstract}
We propose a general class of non-constant volatility models with dependence on the past. The framework includes path-dependent volatility models such as that by Hobson\&Rogers and also path dependent contracts such as options of Asian style. A key feature of the model is that market completeness is preserved. Some empirical analysis, based on the comparison with the performance of standard local volatility and Heston models, shows the effectiveness of the path dependent volatility.
\end{abstract}

\section{Introduction}

In the Black-Merton-Scholes option pricing theory [3], [19], the underlying asset is modeled as a geometric Brownian motion whose dynamic under the risk neutral measure is given by

$$
d S_{t}=r S_{t} d t+\sigma S_{t} d W_{t}
$$

In (1.1) $r$ denotes the locally riskless interest rate and $\sigma$ is the volatility. Under the assumption that both the parameters are constant, model (1.1) leads to closed formulas for plain vanilla options. Nowadays the Black\&Scholes formula is widely used in practice, to the extent that prices of call and put options are usually quoted in terms of the so-called Black\&Scholes implied volatility. However it is also apparent that the prices at which derivatives are traded are inconsistent with the assumption of a constant volatility: indeed especially after the market crash of 1987, the strong empirical evidences of the stochastic nature of the volatility stimulated the development of more realistic models. The overall aim of a non-constant volatility model is twofold: on one hand, to produce prices of plain vanilla options which agree with the observed volatility surfaces and to price exotic options consistently; on the other hand, to find the correct replicating strategy in order to improve the hedging performance.

The first task is usually not difficult to achieve: from a theoretical point of view, any model which depends on a sufficiently large number of parameters can be calibrated to fit (or at least approximate) market prices. But it should be emphasized that any calibration procedure depends on the quantity and quality of the available data: in particular, since only option prices corresponding to a finite number of maturities and strikes are quoted, generally the fitting of prices cannot usually be done in a unique way. Then the essential and hard problem is to determine the "correct" hedging strategy: indeed it is well-known that the hedge parameters are strongly model-dependent even for call and put options (cf. for instance [7]).

From this point of view, the widely used local volatility (henceforth LV) models give poor results. In a LV model the volatility is supposed to be a deterministic function of the time and current price of the underlying asset. The main advantages are that the market is complete and in principle it is possible to specify the volatility function in such a way that option prices given by

*Piazza di Porta S. Donato 5, 40126 Bologna (Italy). E-mail: pascucci@dm.unibo.it 
the model agree with market prices. On the other hand the empirical study by Dumas, Fleming and Whaley [10] shows that, for hedging purposes, the local volatility underperforms an ad-hoc use of the Black\&Scholes model (which consequently should be preferable for its parsimony). The conclusion in [10] is that, as far as one aims to preserve market completeness, a volatility model depending on the whole past trajectory of the asset (instead of the current price alone) should be investigated.

The first results in this direction were obtained by Hobson and Rogers who proposed in [16] a volatility model defined in terms of the difference between the current price and an exponentially weighted average of past prices. Section 2 is devoted to a brief review of this model. We also discuss the main positive aspects and the possible weak points of the Hobson\&Rogers model.

The aim of this paper is to propose a general notion of path dependent volatility which in our opinion overcomes some of the mathematical and economical problems of the Hobson\&Rogers model. This is done in Section 3 where we also prove some results about the absence of arbitrage and completeness of the market in the framework of PDEs and martingales theories. Then in Section 4 we analyze some suitable transformation of the pricing PDE which seems to be more convenient for the numerical approximation.

In Section 5 the path dependent volatility model is validated against market data and the performance compared with those of standard LV and Heston stochastic volatility models. Specifically our aim is to compare the performance of the hedging strategies produced by the models in different market scenarios. To this end we examine the market reversal in May 2006 and observe that a path dependent volatility generally outperforms LV and Heston models: in particular it turns out that the tracking errors of Heston minimum-variance hedging are up to twice the hedging errors of a path dependent volatility model, especially after sudden market movements.

\section{The Hobson\&Rogers model}

In this section we recall the main features of a simplified version of the Hobson and Rogers model [16]. In a Wiener space with one-dimensional Brownian motion $W$, we denote by $S_{t}$ the stock price and by $M_{t}$ and $D_{t}$ respectively the trend and the deviation processes defined by

$$
M_{t}=\lambda e^{-\lambda t} \int_{-\infty}^{t} e^{\lambda s} Z_{s} d s, \quad \lambda>0
$$

and

$$
D_{t}=Z_{t}-M_{t}
$$

where $Z_{t}=\log \left(e^{-r t} S_{t}\right)$ is the log-discounted price process. The function $e^{\lambda s}$ in $(2.1)$ is called the average weight: the parameter $\lambda$ describes the rate at which past prices are discounted.

Hobson and Rogers assume that $S_{t}$ is an Itô process, solution to the stochastic differential equation (SDE)

$$
d S_{t}=\mu\left(D_{t}\right) S_{t} d t+\sigma\left(D_{t}\right) S_{t} d W_{t}
$$

In (2.2), $\mu$ and $\sigma>0$ are deterministic functions satisfying usual hypotheses in order to guarantee that the system of SDEs (2.1)-(2.2) has a solution. A key feature of the model is that the process $\left(S_{t}, D_{t}\right)$ is Markovian (cf. Lemma 3.1 in [16]). Thus, the price of an option, with maturity $T$, can be obtained in terms of solution to the following Cauchy problem (cf. [13]):

$$
\begin{aligned}
\frac{\sigma^{2}(x-y)}{2 \lambda}\left(\partial_{x x} u-\partial_{x} u\right)+(x-y) \partial_{y} u-\partial_{t} u & =0, & & \text { in } \mathbb{R}^{2} \times[0, \lambda T], \\
u(x, y, 0) & =\left(e^{x}-1\right)^{+} & & \text {for }(x, y) \in \mathbb{R}^{2} .
\end{aligned}
$$


Path dependent volatility models are supported by the empirical evidence about the dependence of the volatility with respect to the deviation $D$ : Figure 1 plots implied volatilities against adjusted log-moneyness for options on the S\&P 500 index in the years 2003-2004. The implied volatilities are grouped by ranges of values of $D$. It is immediate to observe on the figure that implied volatilities increase as the $D$ decreases (see also the empirical analysis in [20], Section 2). This enlightens the well-known negative correlation between volatility and market prices.

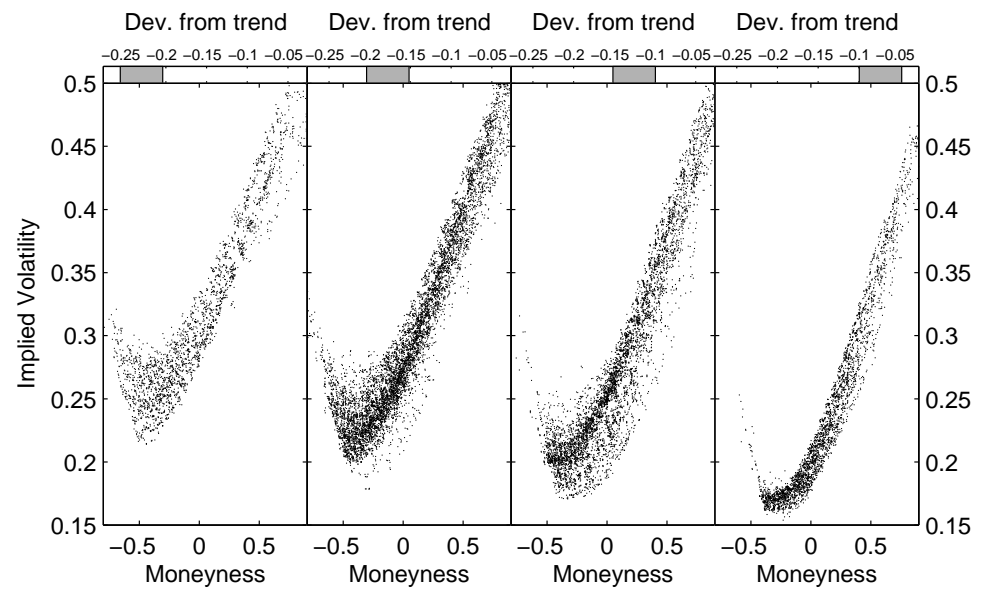

Figure 1: Effects of the deviation from the trend on marked implied volatilities. The implied volatilities are plotted against adjusted $\log$-moneyness $\log (F / K) / \sqrt{T-t}$ and grouped by different ranges of $D_{t}$ as shown by the bar in the top of each panel. Data from the S\&P 500 index options, years 2003-2004.

We emphasize that no additional source of risk has been added in the Hobson\&Rogers model: therefore, unlike many other non-constant volatility models, the market is complete and the arbitrage argument which underlies the Black\&Scholes theory is preserved. While keeping the market completeness, the Hobson\&Rogers model is able to approximate observed volatility surfaces (see the analysis in [13]).

We also remark that a path dependent volatility incorporates information on the past and then, once it is calibrated to the market, the model somehow "knows" the behaviour of investors in different market circumstances and can also keep into account of the positive or negative trend of the asset. For instance, unlike standard local or stochastic volatility models, in case of a sudden fall of the market a path dependent volatility model is designed to automatically increase the level of volatility in order to undertake the market dynamics in a more natural way. This is the reason why it seems that path dependent volatility models do not need to be continuously re-calibrated (which is a well-known disadvantage of local volatility models) and have better out-of-sample performances (see analysis in [13]).

Thanks to these fine features, the Hobson\&Rogers model raised some interest mainly among academics: the problem of parameters calibration ( $\lambda$ in the average weight and the volatility function $\sigma$ ) was studied by Platania and Rogers [20], Figà-Talamanca and Guerra [12] and us in [13]. Di Francesco and one of the authors studied the numerics of the model: we explicitly remark that the PDE in (2.3) is not uniformly parabolic even if it is hypoelliptic by Hörmander's theorem [17]. In particular the convergence of finite difference schemes does not follow by standard arguments but it is proved in [8] as a consequence of some a priori estimates for solutions to (2.3)-(2.4) provided in [9].

An extension to the framework of term-structure modeling was given by Chiarella and Kwon [6]; Hahn, Putschögl and Sass [14] considered the Hobson\&Rogers dynamic in a portfolio optimization problem. Hubalek, Teichmann and Tompkins [18] proposed a generalization to better fit market 
smiles. The robustness of the Hobson\&Rogers model with respect to the data and parameters was studied by Hallulli and Vargiolu [4].

Next we mention some of the weak points of the Hobson\&Rogers model. As noted in [4], some mathematical and economical concerns arise from the definition of the deviation process $D$ in (2.1). Indeed $D$ involves the path of the underlying asset on all its past $]-\infty, t[$. The requirement of an infinite horizon in the past obviously raises practical problems since only finite time series are available so that misspecifications in the model are unavoidable. To overcome this problem, in [4] the following extension of the Hobson\&Rogers model has been proposed: the volatility is specified as

$$
\sigma\left(S_{t}\right)=\sigma\left(S_{t}, Y_{t}, S_{t-\tau}\right)
$$

where

$$
Y_{t}=\int_{t-\tau}^{t} e^{-\lambda(t-v)} f\left(S_{v}\right) d v
$$

where $f$ is a strictly monotone function and $\tau$ is a given delay parameter. Unfortunately the conclusion in [4] is that if $\tau$ is finite then the previous model cannot admit a Markovian realization so that it loses any appeal.

As a further remark, it seems that the average weight $\lambda e^{-\lambda t}$ in (2.1) is not flexible enough to take into account of the special properties of the underlying process that may arise, for instance, from stagionality effects, fusions of stocks, capitalization changes.

In this paper we focus on these problems: we propose a simple generalization of the Hobson\&Rogers model and introduce a new class of models for asset prices with volatility dependent on the past. Our idea is to consider a more flexible deviation process defined in terms of a generic average weight, possibly corresponding to a finite time horizon. We call this the path dependent volatility (henceforth PDV) model. The notion of PDV model is sufficiently general to include the Hobson\&Rogers model and also path dependent derivatives such as Asian style options.

We conclude this section with a further remark. In a PDV model the market is complete since no new sources of randomness are introduced: then we have the apparent advantage that there are unique preference-independent prices for contingent claims. The drawback is that market completeness is barely considered a realistic assumption. In a PDV model options are in principle redundant in that they can be perfectly replicated by delta-hedging in the underlying asset. Then in this framework trading strategies that hedge against volatility risk (for instance, vega-hedging using traded options) are meaningless from a theoretical point of view. An obvious idea is to investigate jump-diffusion or truly stochastic volatility models in the framework of PDV and we aim to come back to this point in a forthcoming paper.

\section{Path dependent volatility}

In order to introduce the PDV model, we consider an average weight $\varphi$ which is a non-negative, piecewise continuous and integrable function on $]-\infty, T]$. We also assume that $\varphi$ is strictly positive in $[0, T]$ and we set

$$
\Phi(t)=\int_{-\infty}^{t} \varphi(s) d s
$$

Then we define the average process as

$$
\left.\left.M_{t}=\frac{1}{\Phi(t)} \int_{-\infty}^{t} \varphi(s) Z_{s} d s, \quad t \in\right] 0, T\right]
$$


where $Z_{t}=\log \left(e^{-r t} S_{t}\right)$ denotes the log-discounted price process. Typical specifications of the average weights are given by following examples:

- $\varphi(t)=e^{P(t)} \max \{Q(t), 0\}$ where $P, Q$ are suitable polynomial functions: the Hobson\&Rogers model corresponds to $P(t)=\lambda t$ and $Q(t)=1$;

- $\varphi(t)=1$ for $t \in[0, T]$ and null elsewhere: this corresponds to the geometric average of an Asian option;

- $\varphi$ piecewise linear function.

We remark that

$$
d M_{t}=\frac{\varphi(t)}{\Phi(t)}\left(Z_{t}-M_{t}\right) d t
$$

then by assuming the following dynamic for the asset log-price

$$
d Z_{t}=\mu\left(Z_{t}-M_{t}\right) d t+\sigma\left(Z_{t}-M_{t}\right) d W_{t},
$$

we deduce that, under standard hypotheses on the coefficients $\mu, \sigma$, the couples $(Z, M)$ and $(Z, D)$ are Markovian processes. Actually in the sequel we restrict ourselves to a Markovian setting and we denote by

$$
V_{t}=\alpha\left(t, Z_{t}, M_{t}\right) S_{t}+\beta\left(t, Z_{t}, M_{t}\right) B_{t}
$$

the value of a portfolio, where $\alpha, \beta$ are suitably regular (deterministic) functions and $B_{t}=e^{r t}$. Moreover we set

$$
f\left(t, Z_{t}, M_{t}\right)=B_{t}^{-1} V_{t}
$$

the discounted value of $V$. The next theorem characterizes the self-financing strategies.

Theorem 3.1. The following conditions are equivalent:

1. the portfolio in (3.3) is self-financing;

2. the function $f$ in (3.4) solves the partial differential equation

$$
\frac{\sigma^{2}(z-m)}{2}\left(\partial_{z z} f-\partial_{z} f\right)+\frac{\varphi(t)}{\Phi(t)}(z-m) \partial_{m} f+\partial_{t} f=0
$$

in $] 0, T\left[\times \mathbb{R}^{2}\right.$, and the following relations hold

$$
\alpha(t, z, m)=e^{-z} \partial_{z} f(t, z, m), \quad \beta(t, z, m)=f(t, z, m)-e^{-z} \partial_{z} f(t, z, m) .
$$

Proof. By the self-financing condition, it holds

$$
d f=-r f d t+B_{t}^{-1}\left(\alpha d S_{t}+\beta d B_{t}\right)=
$$

$\left(\right.$ since $\left.\beta d B_{t}=r \beta B_{t} d t=r\left(V_{t}-\alpha S_{t}\right) d t\right)$

$$
=\alpha B_{t}^{-1}\left(d S_{t}-r S_{t} d t\right)=
$$


(since, by Itô formula, $d S_{t}=B_{t} e^{Z_{t}}\left(d Z_{t}+\left(r+\frac{\sigma^{2}}{2}\right) d t\right)$ )

$$
=\alpha e^{Z_{t}}\left(d Z_{t}+\frac{\sigma^{2}}{2} d t\right) .
$$

On the other hand, by Itô formula and (3.1), we get

$$
d f\left(t, Z_{t}, M_{t}\right)=\left(\partial_{t} f+\frac{\sigma^{2}}{2} \partial_{z z} f+\frac{\varphi}{\Phi}\left(Z_{t}-M_{t}\right) \partial_{m} f\right)+\partial_{z} f d Z_{t} .
$$

Comparing (3.7) and (3.8), by the uniqueness of the representation of an Itô process, we infer

$$
\partial_{z} f\left(t, Z_{t}, M_{t}\right)=\alpha\left(t, Z_{t}, M_{t}\right) e^{Z_{t}}
$$

Now we recall that, since by assumption $\varphi$ is strictly positive on $[0, T]$, the conditions of the classical Hörmander's theorem are satisfied and the process $\left(Z_{t}, M_{t}\right)$ has a strictly positive density on $\mathbb{R}^{2}$ for $t>0$ (we also refer to the paper [9] by Di Francesco and one of the authors for a direct proof of this result). Then (3.6) readily follows from (3.9). Analogously, by equating the $d t$-parts of (3.7) and (3.8) and using (3.9), we obtain the PDE (3.5).

We do not prove the inverse implication which is straightforward.

Next we prove that in the PDV model the market is arbitrage-free and complete.

Corollary 3.2. For any contingent claim $H=H\left(S_{T}, M_{T}\right)$, with $H \in L_{\text {loc }}^{1}\left(\mathbb{R}^{2}\right)$ and $H \geq 0$, there exists a unique self-financing and admissible ${ }^{1}$ strategy replicating $H$. The strategy is determined by formulas (3.6) where $f$ is the unique solution of the Cauchy problem for equation (3.5) with final condition

$$
f(T, z, m)=e^{-r T} H\left(e^{z}, m\right) .
$$

In particular, the market is arbitrage-free and complete: $H_{t}:=e^{r t} f\left(t, Z_{t}, M_{t}\right)$ is the arbitrage price of the claim $H$.

Proof. The thesis is a direct consequence of Theorem 3.1 and of the existence and uniqueness results for degenerate parabolic equations of Kolmogorov type (which include (3.5)) proved by Di Francesco and one of the authors [9] and by Polidoro [21].

The previous results can be also proved by using the martingale theory. Note that the dynamic of the stock price $S_{t}$ is given by

$$
d S_{t}=\left(r+\mu\left(D_{t}\right)+\frac{\sigma^{2}\left(D_{t}\right)}{2} S_{t}\right) d t+\sigma\left(D_{t}\right) S_{t} d W_{t}
$$

where the deviation process $D_{t}=Z_{t}-M_{t}$ satisfies the SDE

$$
d D_{t}=\left(\mu\left(D_{t}\right)-\frac{\varphi}{\Phi} D_{t}\right) d t+\sigma\left(D_{t}\right) d W_{t} .
$$

Then we set

$$
\theta\left(D_{t}\right)=\frac{\sigma\left(D_{t}\right)}{2}+\frac{\mu\left(D_{t}\right)}{\sigma\left(D_{t}\right)}
$$

\footnotetext{
${ }^{1}$ Such that the value of the portfolio is non-negative.
} 
and consider the process

$$
\widetilde{W}_{t}=W_{t}+\int_{0}^{t} \theta\left(D_{s}\right) d s
$$

Under suitable conditions on the coefficients (cf. for instance the Appendix in [16]) the position

$$
\frac{d Q}{d P}=\exp \left(-\frac{1}{2} \int_{0}^{t} \theta^{2}\left(D_{s}\right) d s-\int_{0}^{t} \theta\left(D_{s}\right) d W_{s}\right)
$$

defines a probability measure $Q$ on the filtration $\mathcal{F}_{t}$ of $W$, which is equivalent to $P$ and such that $\widetilde{W}$ is a $Q$-Brownian motion. Then under $Q$ we have

$$
d S_{t}=r S_{t} d t+\sigma\left(D_{t}\right) S_{t} d \widetilde{W}_{t}
$$

so that the discounted price $e^{-r t} S_{t}$ is a $Q$-martingale and the arbitrage price of a contingent claim $H$ can be written

$$
H_{t}=e^{-r(T-t)} E^{Q}\left(H \mid \mathcal{F}_{t}\right) .
$$

\section{Some convenient transformation}

For an European call with strike $K$, the pricing PDE (3.5) is coupled with the final condition

$$
f(T, z, m)=e^{-r T}\left(e^{z}-K\right)^{+} .
$$

By the change of variables

$$
f(t, z, m)=K u(T-t, z-\log K, m-\log K)
$$

we obtain the equivalent Cauchy problem

$$
\begin{array}{ll}
\frac{\sigma^{2}(x-y)}{2}\left(\partial_{x x} u-\partial_{x} u\right)+\frac{\varphi(T-\tau)}{\Phi(T-\tau)}(x-y) \partial_{y} u-\partial_{\tau} u=0, & \tau \in] 0, T\left[, \quad(x, y) \in \mathbb{R}^{2},\right. \\
u(0, x, y)=e^{-r T}\left(e^{x}-1\right)^{+}, & x \in \mathbb{R} .
\end{array}
$$

Note that problem (4.2)-(4.3) is independent of $K$ and therefore it allows to price all call options with different strikes and maturities in a single run.

In view of the numerical approximation, we also consider the following further change of variables:

$$
\tau=g(\tau):=-\log \Phi(T-\tau)
$$

If $u(\tau, x, y)=v(g(\tau), x, y)$ then

$$
\partial_{\tau} u=\frac{\varphi(T-\tau)}{\Phi(T-\tau)} \partial_{t} v
$$

and equation (4.2) is equivalent to

$$
a(x-y, t)\left(\partial_{x x} v-\partial_{x} v\right)+(x-y) \partial_{y} v-\partial_{t} v=0,
$$


for $t \in]-\log \Phi(T),-\log \Phi(0)\left[\right.$ and $(x, y) \in \mathbb{R}^{2}$, where

$$
a(x-y, t)=\frac{\sigma^{2}(x-y)}{2 \frac{\varphi}{\Phi}\left(g^{-1}(t)\right)} .
$$

For instance, in the Hobson\&Rogers model, $\frac{\varphi}{\Phi} \equiv \lambda$ and $g(t)=\lambda(t-T)$ so that the PDE has to be solved for $t \in[-\lambda T, 0]$.

It is clear that in the case of constant volatility function $\sigma$ in (3.2), the model reduces to the classical Black\&Scholes framework independently of $\varphi$. In the case of an Asian option, the following change of variables

$$
f(t, x, \eta)=u\left(t, x, \frac{\eta}{\Phi(T-t)}\right), \quad y=\frac{\eta}{\Phi(T-t)}
$$

seems to be convenient. Indeed we have

$$
\partial_{\eta} f=\frac{1}{\Phi(T-t)} \partial_{y} u, \quad \partial_{t} f=\frac{\varphi(T-t)}{\Phi^{2}(T-t)} \eta \partial_{y} u+\partial_{t} u,
$$

and therefore $u$ is solution to (4.2) if and only if

$$
\left.\frac{\sigma^{2}}{2}\left(\partial_{x x} f-\partial_{x} f\right)+\varphi(T-\tau) x \partial_{\eta} f-\partial_{t} f=0, \quad t \in\right] 0, T\left[, \quad(x, y) \in \mathbb{R}^{2}\right.
$$

Note that $\varphi \equiv 1$ for a geometric average Asian option. We also remark that the explicit expression of the fundamental solution to equation (4.5), even for a generic $\varphi$, is known (cf. Barucci, Polidoro and Vespri [2]).

\section{$5 \quad$ Empirical tests}

In this section the PDV model is calibrated to real market data and compared with some standard non-constant volatility models, namely: the standard Hobson\&Rogers [16], Dupire LV [11] and Heston [15] stochastic volatility models.

We begin by defining the weight function $\varphi(t)$ in terms of $g^{\prime}(t)=\varphi(t) / \Phi(t)$ (using the notation introduced in Section 4). In particular we choose $g^{\prime}(t)$ as a piecewise linear function defined by

$$
g^{\prime}(t)=\sum_{i=0}^{K} \alpha_{i} s_{i}(t)
$$

where

$$
\begin{array}{rlr}
s_{0}(t) & =\widetilde{s}(t / \delta) \chi_{[0, \delta)}+\chi_{(-\infty, 0)}, & \\
s_{K}(t) & =\widetilde{s}(t / \delta-K) \chi_{[T-\delta, T)}+\chi_{[T, \infty)}, & \\
s_{i}(t) & =\widetilde{s}(t / \delta-i), & \text { for } i=2, \ldots, K-1
\end{array}
$$

with $\delta=T / K$ and

$$
\widetilde{s}(t)=(t+1) \chi_{[-1,0)}+(1-t) \chi_{[0,1)} .
$$

That is, $s_{i}(t)(i=2, \ldots, K-1)$ are the hat functions centered at $i \delta$ with support $[(i-1) \delta,(i+1) \delta]$, $s_{1}(t)$ and $s_{K}(t)$ are such that $g^{\prime}(t)$ is constant outside $[0, T]$ as shown in Figure 2. 


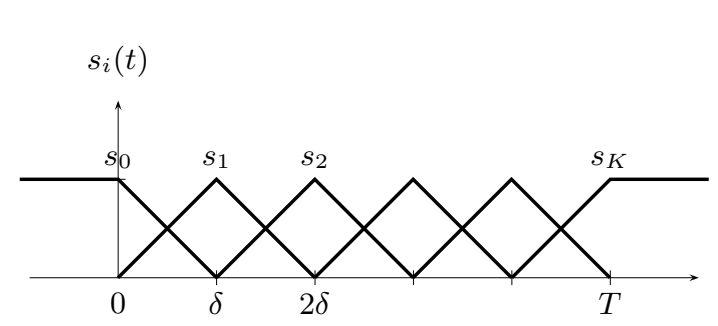

(a)

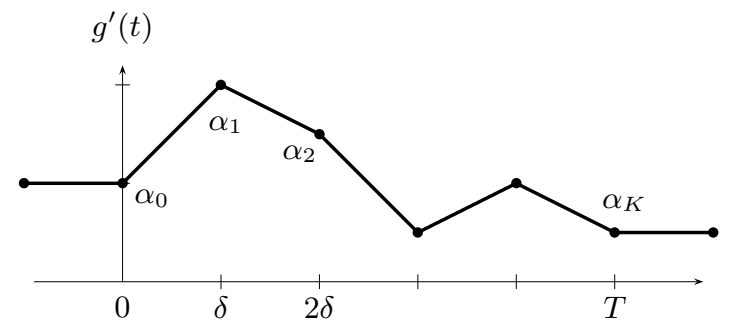

(b)

Figure 2: The hat functions $s_{i}(t)$ for $i=1, \ldots, K$ and $g^{\prime}(t)$

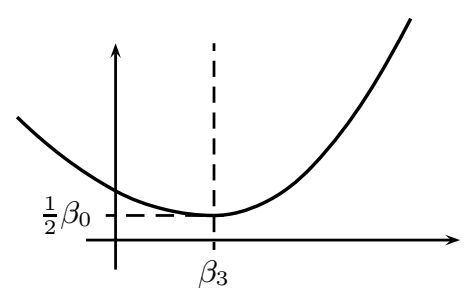

Figure 3: The $\sigma^{2}$ function for $\beta_{0}=1 / 2, \beta_{1}=1, \beta_{2}=1 / 2$ and $\beta_{3}=1$,.

The volatility function is defined as

$$
\sigma^{2}(d)= \begin{cases}\frac{1}{2} \max \left(\sigma_{\text {min }}^{2}+\alpha_{r}\left(d-d_{0}\right)^{2}, 2 \sigma_{\text {Max }}^{2}\right), & \text { if } d \geq d_{0} \\ \frac{1}{2} \max \left(\sigma_{\text {min }}^{2}+\alpha_{l}\left(d-d_{0}\right)^{2}, 2 \sigma_{\text {Max }}^{2}\right), & \text { if } d<d_{0} .\end{cases}
$$

An example of $\sigma$ is shown in Figure 3. Overall, the number of parameters to be calibrated is $K+5$ : $\sigma_{\text {min }}^{2}, \alpha_{l}, \alpha_{r}, d_{0}$ to specify the volatility function $\sigma$, and $\alpha_{0}, \alpha_{1}, \ldots, \alpha_{K}$ to specify the weight $\varphi$.

We use the approach proposed in [13] which allows to price options of different strikes and different times-to-expiration by a single numerical solution of the Cauchy problem (4.2)-(4.3).

The Hobson\&Rogers model used in the comparison is defined by the SDEs (2.1) and (2.2) where $\sigma$ is specified in (5.1).

In the LV model, $S_{t}$ is solution of the SDE

$$
d S_{t}=\mu_{t} S_{t} d t+\sigma\left(S_{t}, t\right) S_{t} d W_{t} .
$$

As shown by Dupire [11], the LV function $\sigma\left(S_{t}, t\right)$ can be directly computed by knowing the option price as a function of strike and maturity. The number of parameters we have used is equal to the number of option prices in the cross section.

In the stochastic volatility model by Heston, $S_{t}$ and $\sigma_{t}^{2}$, the price and the squared volatility processes, respectively, are given, in the risk neutral measure, by the solution of the SDE

$$
\begin{aligned}
& d S_{t}=r_{t} S_{t} d t+\sigma_{t} S_{t} d \hat{W}_{t} \\
& d \sigma_{t}^{2}=\kappa\left(\sigma_{\infty}^{2}-\sigma_{t}^{2}\right) d t+\gamma \sigma_{t}\left(\rho d \hat{W}_{t}+\sqrt{1-\rho^{2}} d \hat{W}_{t}\right)
\end{aligned}
$$

where $d \hat{W}_{t}$ and $d \hat{\hat{W}}_{t}$ are two independent Brownian motions on the risk-neutral probability measure. The approach used to compute the prices of European options is the computational method of Carr 
and Madan which uses a Fourier inversion technique $[15,5]$. In the experiments, the $\sigma_{\infty}$, the longterm volatility, $\kappa$, the mean reversion speed, $\gamma$, the volatility of volatility, and $\rho$, the correlation, are inferred from market prices.

\subsection{Empirical results}

The dataset consists in closing prices of options on futures on the FTSE-100 index quoted at Euronext in the period March 22 - May 19, 2006 and maturities on June, September, December 2006 and March, June, September and December 2007. For each day and each maturity the dataset contains the underlying future price (with values in the range [5675,6307]), the Call and Put closing prices for strikes 4025-6725 and the corresponding implied volatilities. The underlying values have been corrected for dividends, in order to have a common underlying for all the expirations and then option prices are recomputed by using the dataset's implied volatilities. Thus, after the adjustment the underlying has a null drift in the equivalent martingale measure, that is the interest and dividend rates are null. An example of the implied volatility surface is shown in Figure 4

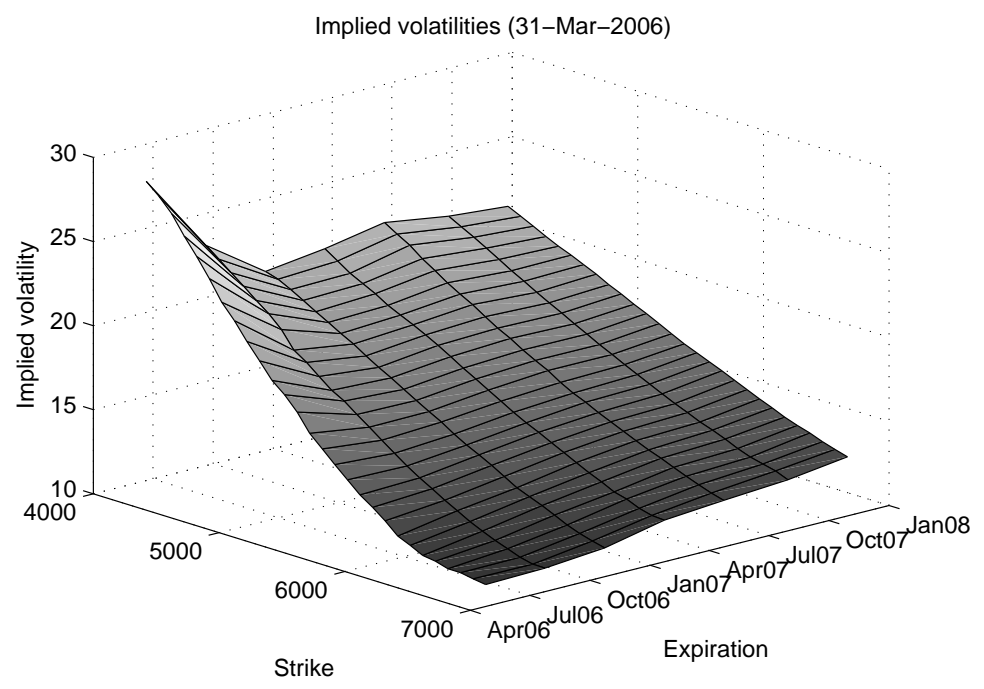

Figure 4: Implied Volatility surface for March 31, 2006.

In the first set of experiments, the parameters of the four models are daily calibrated to market prices by a least squares fitting of market prices as in $[10,13]$. An example of the absolute pricing errors of the four models on a specific date is reported in Figure 5.

A resume of the performances on each are reported in Figures 6 and 7. These figures plot for each day and for each model the Residual Mean Squared Errors (RMSE) and Residual Mean Squared percentage errors (RMSPE). In the computation of the RMSPE options with price smaller than 5 have been discarded. As can be seen from Figure 6 the fitting of the LV model is, as expected, always almost perfect; that of standard Hobson\&Rogers model is at least twice that of the remaining two models. The Heston model is slightly better than the PDV; however the reverse happen when considering relative errors (cf. Figure 7).

In order to study the stability of the parameters on different samples, their evolution is reported in Figures 8-10 for the Hobson\&Rogers, Heston and PDV models. Due to its large number we do not report the evolution of Dupire's local volatilities, but it is well known they are not stable due to its over-parametrization. The parameters for the Hobson\&Rogers model are quite stable until near the end of the sample, where the model flattens to standard Black and Scholes, $\alpha_{l}, \alpha_{r} \simeq 0$. This change of regime happens also for the Heston and PDV models, but a bit more in advance. 


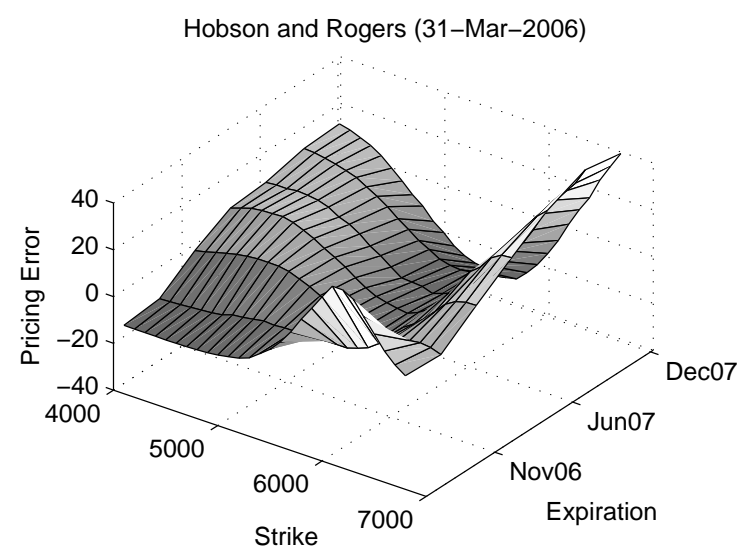

(a) Hobson\&Rogers errors

Path Dependent Volatility (31-Mar-2006)

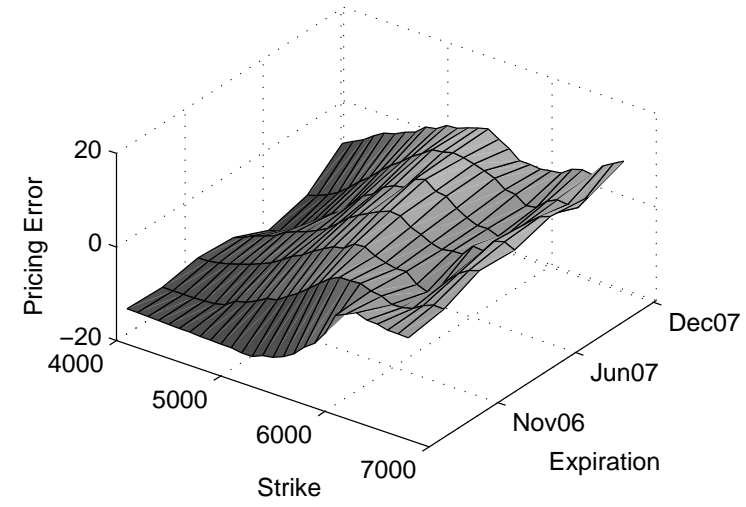

(c) PDV errors

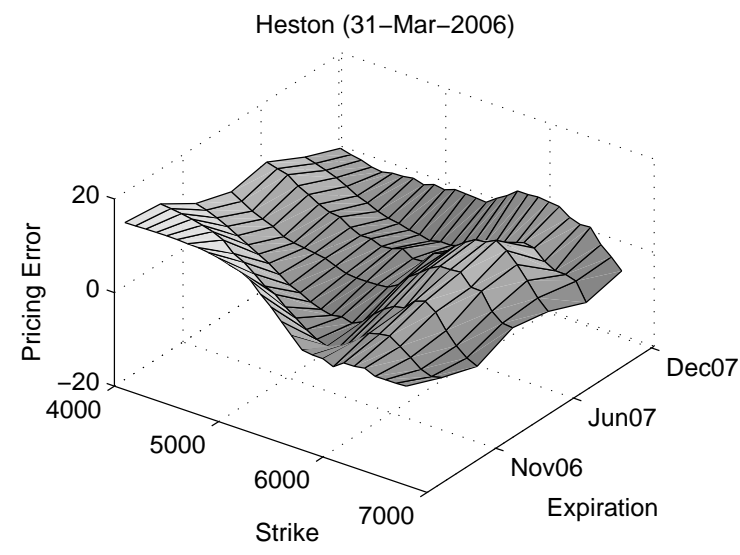

(b) Heston errors

Dupire (31-Mar-2006)

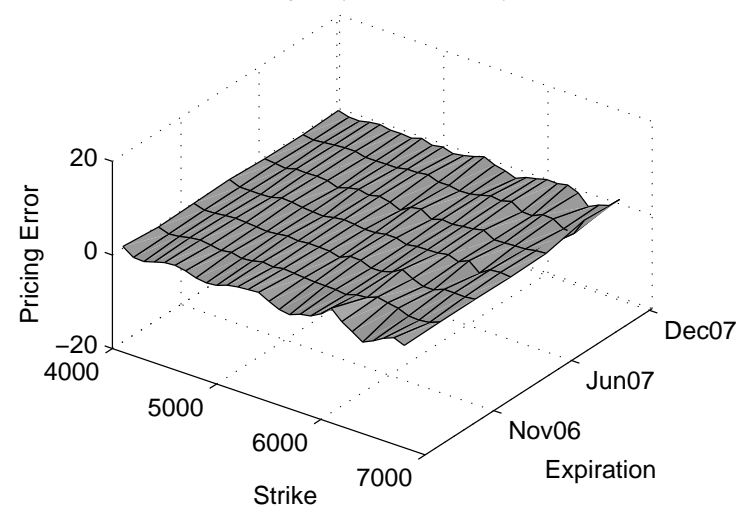

(d) LV's errors

Figure 5: Pricing error surfaces for Hobson\&Rogers, Heston, PDV and Dupire models on March 31, 2006. Value of the underlying is 5964.5 .

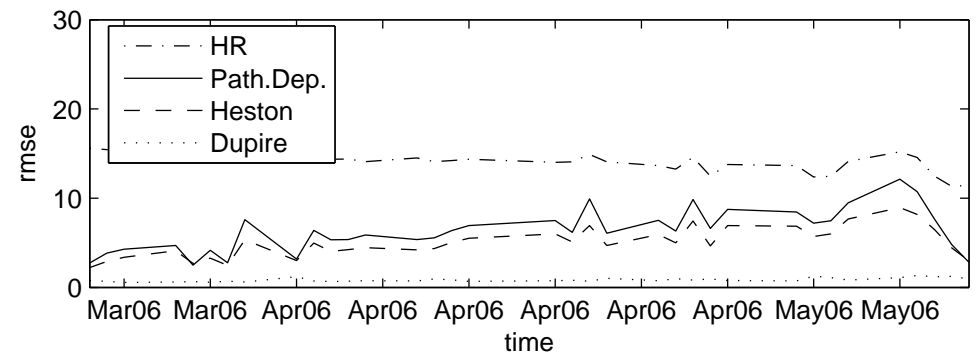

Figure 6: Root Mean Squared pricing Errors of for each day in the test period. 


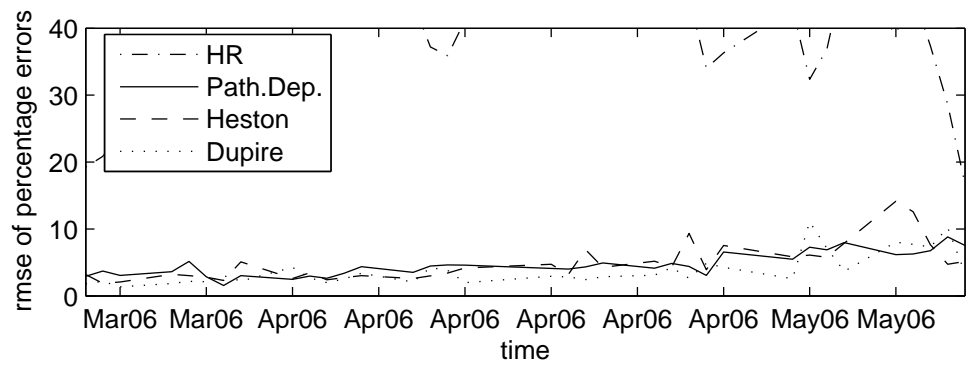

Figure 7: Root Mean Square of Percentage Errors in pricing.

These two models show spikes in the series of parameters on the same day (cf. the graph of $\rho$ in Figure 9 and of $\alpha_{r}$ in Figure 10).

This behaviour of the three models can be explained by looking at the time series of the underlying index which is shown in Figure 11. Near the end of the sample, exactly on May 12, the index level drops significantly: consequently the option market reacts and the parameters of the three models try to adapt to market movements.

As a final (and more significant) experiment we compared the hedging performances of the methods by considering the tracking error of the replicating portfolio suggested by each model w.r.t. the evolution of each single call.

For each model we proceed as follows. At the $i$ th day, time $t_{i}$, the model has been calibrated to the market cross-section of prices. Then, for a given expiry $T$ and a given strike $K$ we consider the portfolio composed by a short position on one Call $C_{t_{i}}$ and a long on the replicating portfolio $V_{t_{i}}=\alpha_{i} S_{t_{i}}+\beta_{i} B_{t_{i}}$ as in (3.3). That is, the portfolio $\Pi_{t_{i}}=V_{t_{i}}-C_{t_{i}}$, which has null value in case of perfect replication. Then, the next day the portfolio has value given by $\alpha_{i} S_{t_{i+1}}+\beta_{i} B_{t_{i+1}}-C_{t_{i+1}}$, the corresponding profit and losses are accumulated and a new portfolio $\Pi_{t_{i+1}}$ is built. Recalling that we are working on a dividend and interest rate free setting, the total profits and losses on the period $\left[t_{1}, t_{n}\right]$ are given by $C_{1}-C_{n}+\sum_{i=1}^{n-1} \alpha_{i}\left(S_{t_{i+1}}-S_{t_{i}}\right)$.

This procedure has been repeated for each model, for each strike, for the expirations June 16, 2006 and December 15, 2006 and for the periods March 22 - May 5 and March 22 - May 19. The two periods correspond to quiet and nervous market situations, respectively (see Figure 11). These performance results are reported on Figures 12-15. Standard delta-hedging have been used for HR and PDV models, while minimum-variance hedging is used for Heston stochastic volatility model $[1]$.

Figure 12 shows the replication error of the hedging strategies until about one month to expiration and after the fall of May 12. The HR model is the better for at-the-money options and the Heston model is superior for out-of-the-money options. The PDV model is between the two, but in both the cases is near to the best and the overall performance is thus preferable to the other two.

The performances in a quiet market scenario reported in Figure 13 are a bit mixed. In this experiment the overall performances of Heston are slightly better than those of PDV and HR models.

Similar, but less marked, results are reported in Figures 14 and 15 where far from expiration options are considered. In the nervous scenario the HR model is more protective trying to limiting the losses, Heston model is not able to do that and the PDV model lies between the two. On the contrary, in the quiet scenario, Heston has a slightly better performance but the difference between the three models is not too strong. 

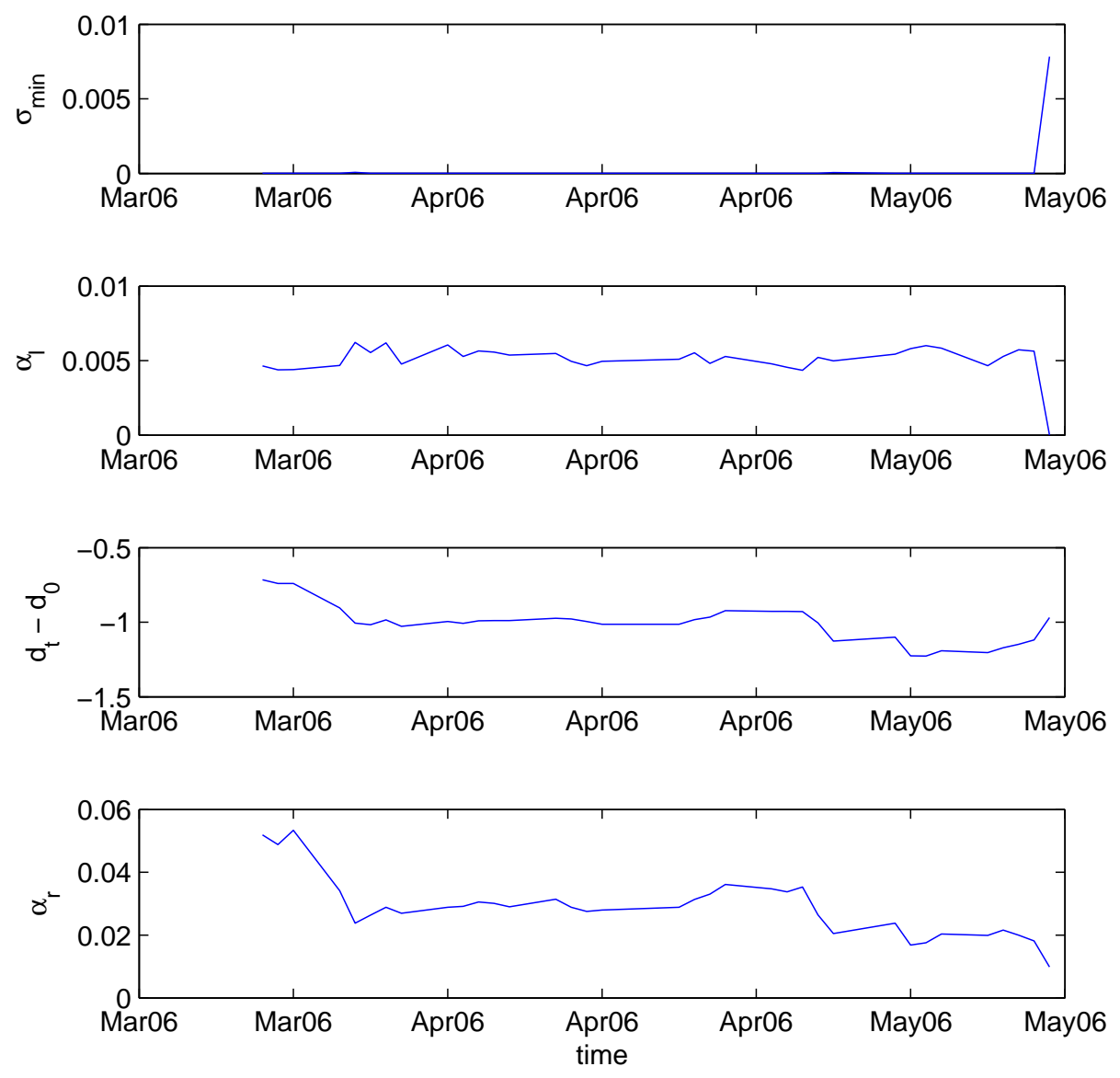

Figure 8: Evolution of parameters for HR model, with daily calibration on the test period (March 22 - May 19, 2006). 

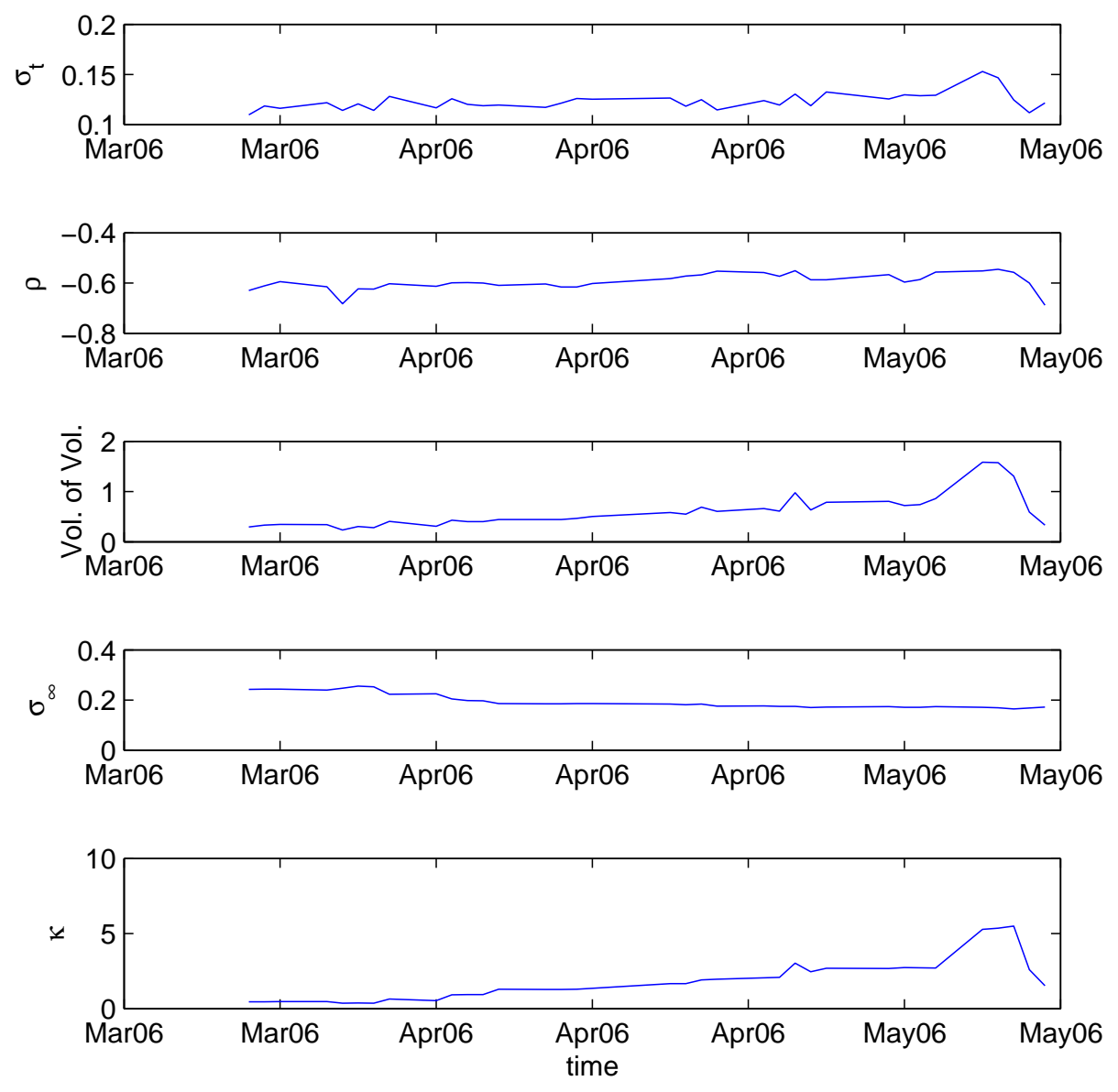

Figure 9: Evolution of Heston parameters with daily calibration on the test period. 

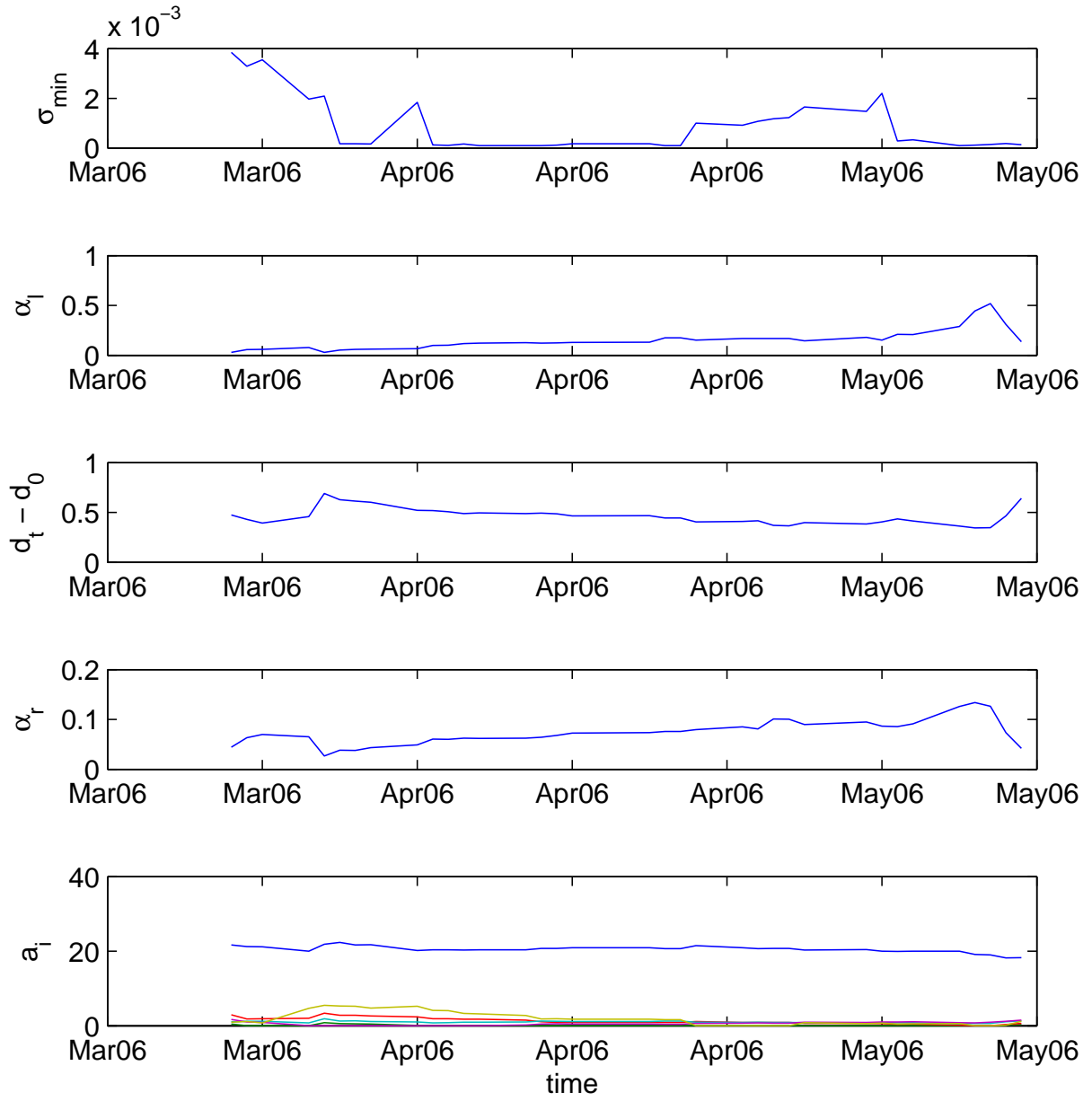

Figure 10: Evolution of parameters for the PDV model, with daily calibration on the test period.

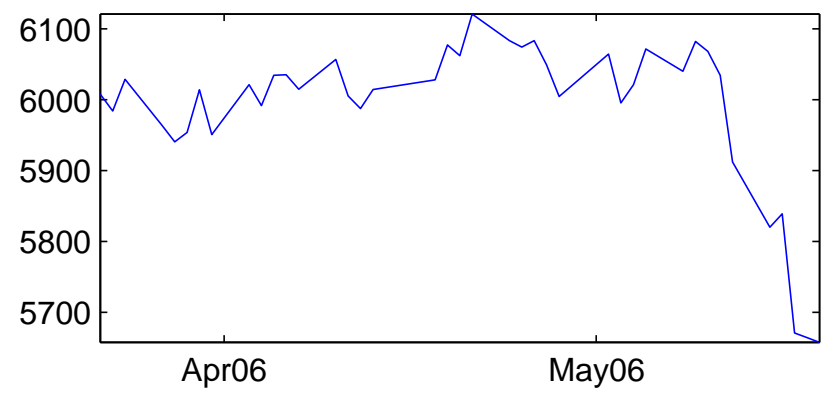

Figure 11: Closing prices for the FTSE-100 index on the period March 22 - May 19, 2006. . 


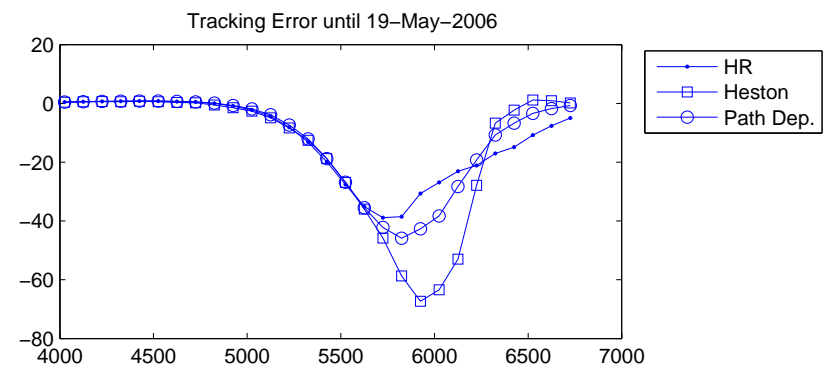

Figure 12: Hedging error against strike for maturity June 16, 2006. The errors are computed on the period March 22 - May 19, 2006.

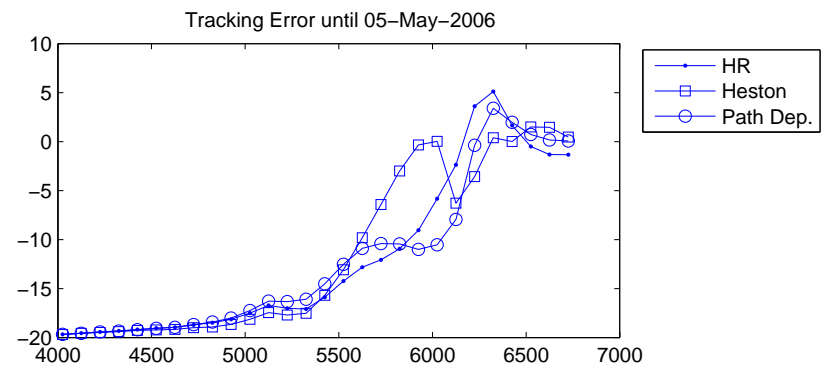

Figure 13: Hedging error against strike for maturity date June 16, 2006. The errors are computed on the period March 22 - May 5, 2006.

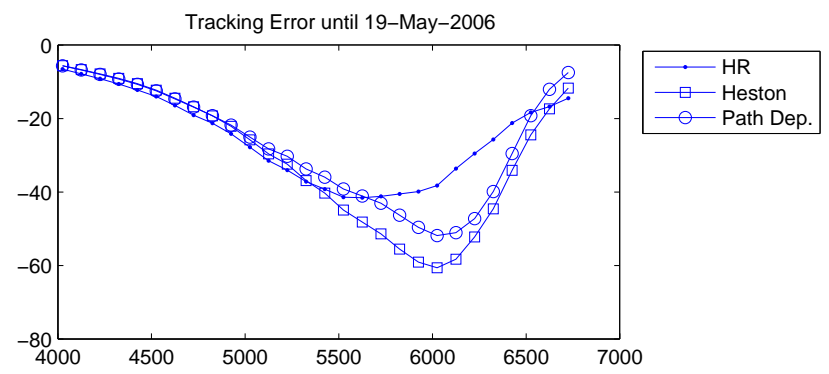

Figure 14: Hedging error against strike for maturity December 15, 2006. The errors are computed on the periodt March 22 - May 19, 2006.

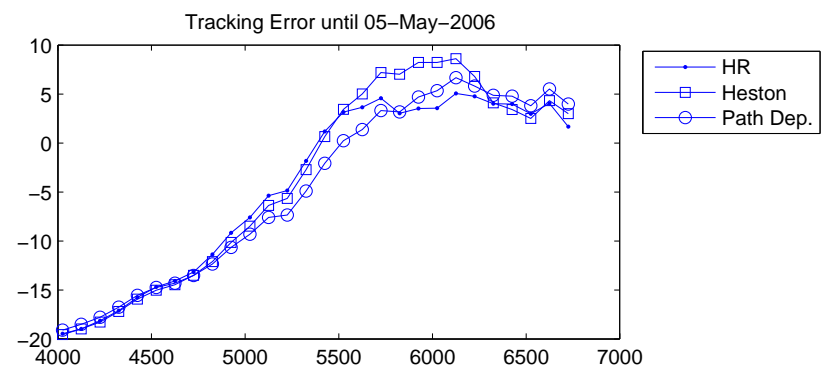

Figure 15: Hedging error against strike for maturity December 15, 2006. The errors are computed on the periodt March 22 - May 5, 2006. 


\section{References}

[1] C. Alexander and L. Nogueira, Hedging options with scale-invariant models, tech. report, ICMA Centre, University of REading, June 2006.

[2] E. Barucci, S. Polidoro, And V. Vespri, Some results on partial differential equations and Asian options, Math. Models Methods Appl. Sci., 11 (2001), pp. 475-497.

[3] F. Black and M. Scholes, The pricing of options and corporate liabilities, J. Political Economy, 81 (1973), pp. 637-654.

[4] V. Blaka Hallulli and T. Vargiolu, Financial models with dependence on the past: a survey, Applied and Industrial Mathematics in Italy, M. Primicerio, R. Spigler, V. Valente, editors, Series on Advances in Mathematics for Applied Sciences, World Scientific 2005, 69 (2005).

[5] P. CARR And D. Madan, Option pricing and the fast fourier transform, Journal of Computational Finance, 2 (1999), pp. 61-73.

[6] C. Chiarella And K. Kwon, A complete Markovian stochastic volatility model in the HJM framework, Asia-Pacific Financial Markets, 7 (2000), pp. 293-304.

[7] R. Cont, Model uncertainty and its impact on the pricing of derivative instruments, Math. Finance, 16 (2006), pp. 519-547.

[8] M. Di Francesco And A. Pascucci, On the complete model with stochastic volatility by Hobson and Rogers, Proc. R. Soc. Lond. Ser. A Math. Phys. Eng. Sci., 460 (2004), pp. 33273338 .

[9] —, On a class of degenerate parabolic equations of Kolmogorov type, AMRX Appl. Math. Res. Express, (2005), pp. 77-116.

[10] B. Dumas, J. Fleming, and R. E. Whaley, Implied volatility functions: empirical tests, J. Finance, 53 (1998), pp. 2059-2106.

[11] B. DupIRE, Pricing and hedging with smiles, in Mathematics of derivative securities (Cambridge, 1995), vol. 15 of Publ. Newton Inst., Cambridge Univ. Press, Cambridge, 1997, pp. 103111.

[12] G. FigÀ-Talamanca And M. L. Guerra, Complete models with stochastic volatility: further implications, Working Paper, Università della Tuscia, Facoltà di Economia, 5 (2000).

[13] P. Foschi And A. Pascucci, Calibration of the Hobson 8 Rogers model: empirical tests., Preprint AMS Acta, University of Bologna, (2005).

[14] M. Hahn, W. Putschögl, And J. Sass, Portfolio optimization with non-constant volatility and partial information, preprint, (2006).

[15] S. Heston, A closed-form solution for options with stochastic volatility with applications to bond and currency options., Review of Financial Studies, 6 (1993), pp. 327-343.

[16] D. G. Hobson And L. C. G. Rogers, Complete models with stochastic volatility, Math. Finance, 8 (1998), pp. 27-48. 
[17] L. Hörmander, Hypoelliptic second order differential equations, Acta Math., 119 (1967), pp. 147-171.

[18] F. Hubalek, J. Teichmann, and R. Tompkins, Flexible complete models with stochastic volatility generalising Hobson-Rogers, working paper, (2004).

[19] R. C. Merton, Theory of rational option pricing, Bell J. Econom. and Management Sci., 4 (1973), pp. 141-183.

[20] A. Platania and L. C. G. Rogers, Putting the HobsonERogers model to the test, working paper, (2006).

[21] S. Polidoro, Uniqueness and representation theorems for solutions of Kolmogorov-FokkerPlanck equations, Rend. Mat. Appl. (7), 15 (1995), pp. 535-560. 